The NEXT-100 experiment for Neutrino-less Double Beta decay: Main features, Results from Prototypes and Radiopurity issues.

Javier Pérez Pérez ${ }^{1}$

Universidad Autónoma de Madrid

Instituto de Física Teórica UAM/CSIC

28049 Cantoblanco (Madrid), Spain

E-mail: javier.perez.perez@uam.es

\begin{abstract}
:
The NEXT-100 time projection chamber, currently under construction, will search for neutrinoless double beta decay $(2 \beta 0 \mathrm{v})$ using $100 \mathrm{~kg}$ at $15 \mathrm{bar}$ of high-pressure xenon gas enriched in the ${ }^{136} \mathrm{Xe}$ isotope to $90 \%$. It will be running in the Canfranc Underground Laboratory (LSC) under the Pyrenees in Spain. The detector possesses two important features for $2 \beta 0 v$ searches: very good energy resolution (better than $1 \%$ FWHM at the $\mathrm{Q}$ values of the ${ }^{136} \mathrm{Xe}$ ) and event topological information for the distinction between signal and background. Furthermore, the technique can be extrapolated to the ton-scale, thus allowing the full exploration of the inverted hierarchy of neutrino masses. First prototypes have been operating successfully in different laboratories.

I will discuss the main features of NEXT-100 and the prototype results concerning energy resolution, track pattern recognition and xenon gas ionization and scintillation properties obtained with a variety of radioactive sources. We will present as well the main results so far on radioactive contamination of the materials to be used in NEXT-100, including the status of the measurement campaign of its 60 low-radioactivity Photo Multipliers Hamamatsu R11410-10.
\end{abstract}

The European Physical Society Conference on High Energy Physics

18-24 July, 2013

Stockholm, Sweden

\footnotetext{
${ }^{1}$ Speaker
} 


\section{NEXT Detectors}

NEXT-100 [1] will be an asymmetric Time Projection Chambers (TPC) filled with highpressure xenon gas (100 kg of enriched xenon, $90 \%$ of $\left.{ }^{136} \mathrm{Xe}\right)$ at $15 \mathrm{bar}$. It has two different measurement planes: energy plane to perform a precise measure of the energy with a very good energy resolution, $0.5-1 \%$ at $\mathrm{Q}_{\beta \beta}$ and tracking plane to reconstruct the track of the event and do background rejection.
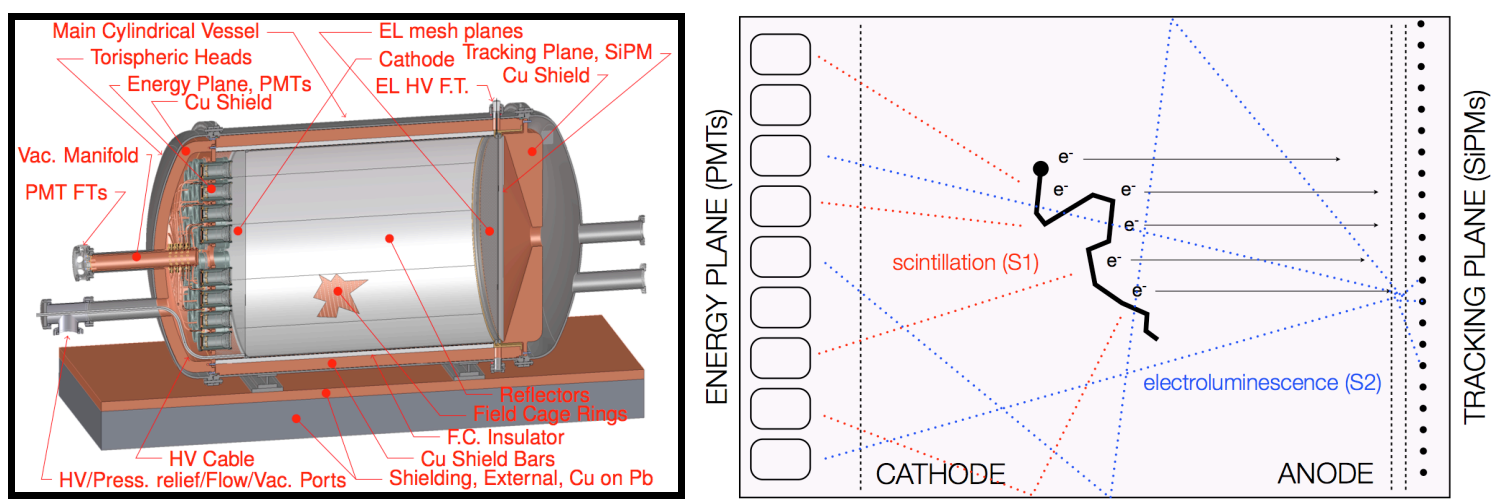

Figure 1. Left: NEXT-100 Detector scheme. Right: light production of an electron track.

1.1 NEXT Conceptual Idea: advantages

NEXT idea has several advantages over the other competitors. The advantages of using Xe are [2]: a high $\mathrm{Q}_{\beta \beta}$, it is a noble gas (the only noble gas with $2 \beta$-decay) and therefore features a low attachment, the high natural abundance of ${ }^{136} \mathrm{Xe}$ isotope and it is easy to enrich. The advantages of using gas Xe are the good energy resolution $(<1 \%)$ and the possibility of tracking. In addition, combining tracking and radiopure selection of the components give us a very low background. Further, those properties make the NEXT concept scalable.

\subsubsection{NEXT Conceptual Idea: light production}

In the detector, the electrons from 2 $\beta$-decay excite and ionize the Xenon gas (Fig. 1, right). The Xenon atoms de-excitate very rapidly ( $\sim \mathrm{ns})$ emitting a rather small $172 \mathrm{~nm}$ light signal (S1), that is detected at the energy plane and serves as trigger. The ionization electrons are drifted by a weak electric field to the electroluminescent (EL) region. There, a larger electric field such to excite the xenon, but not enough to ionize it, accelerates electrons producing a big amount of $172 \mathrm{~nm}$ scintillation light (S2). This light is wave-length-shifted (WLS) to the blue region by a Tetra Phenyl Butadiene (TPB) coated on reflectors that cover a large fraction of the inner part of the field cage. The energy plane will measure precisely the number of photons produced (Fig. 2, left).

\subsubsection{NEXT Conceptual Idea: tracking}

Close to the EL region, we have the tracking plane. There, TPB coated Si-Photomultipliers (SiPMs) reconstruct the two electrons tracks from the $2 \beta$-decay. They form a single twisted line 
with a strong energy deposition at both ends. This technique is crucial to reject background events (Fig. 2, right).
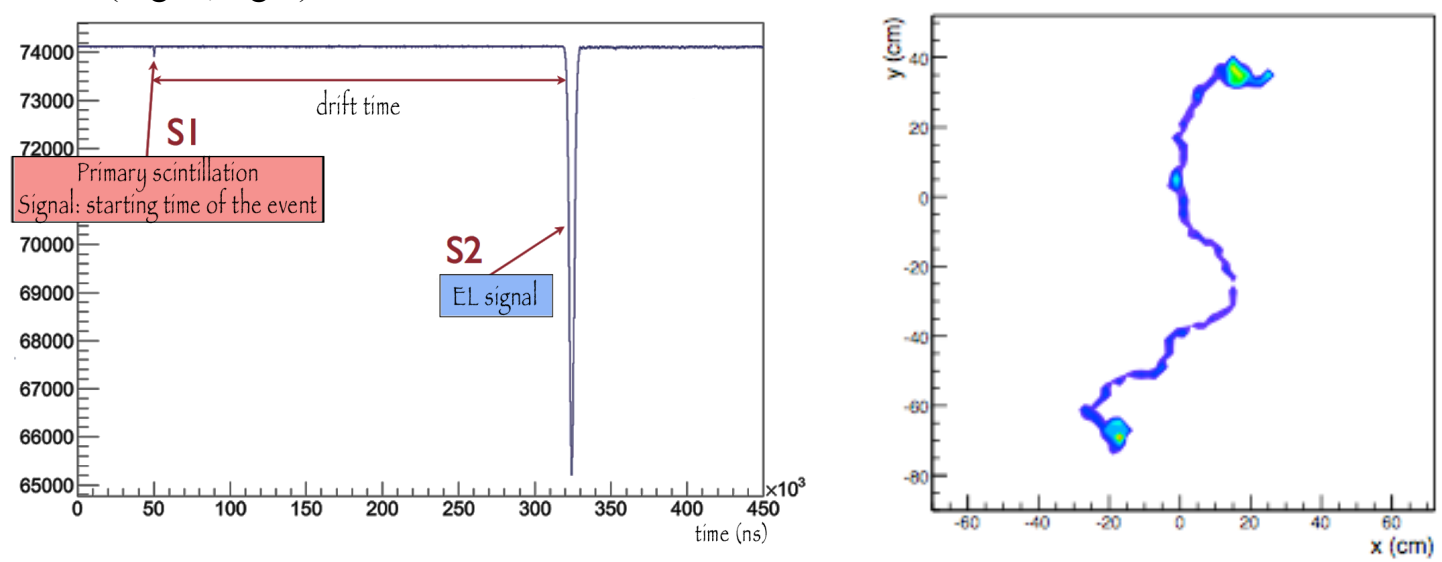

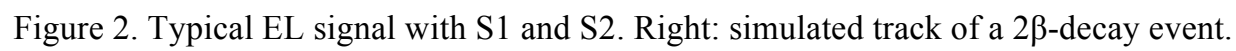

\section{Detectors}

The scalability of the NEXT idea has allowed us to build several prototypes to test the technology as well as to find and fix some of the unforeseen problems. The most important prototypes are NEXT-DEMO and NEXT-DBDM.

\subsection{Prototype NEXT-DEMO}

NEXT-DEMO [3] [4] is a prototype to validate the NEXT-100 design. It is installed in a white room at Instituto de Física Corpuscular (IFIC), in Valencia, Spain. It consist of a highpressure (10 atm, $\left.\rho=5 \cdot 10^{-2} \mathrm{~g} / \mathrm{cm}^{3}\right)$ xenon electroluminescent $\mathrm{TPC}\left(\mathrm{HV}_{\text {cathode }}=25 \mathrm{kV}, \mathrm{HV}_{\text {anode }}=\right.$ $10 \mathrm{kV})$ with cylindrical active volume (30 cm long and $16 \mathrm{~cm}$ diameter) and a TPB coated hexagonal tube made of panels of PTFE, with nearly 100\% diffuse reflectivity. It has 19 Hamamatsu R7378A PMTs (energy plane) and 256 TPB coated SiPMs (tracking plane).
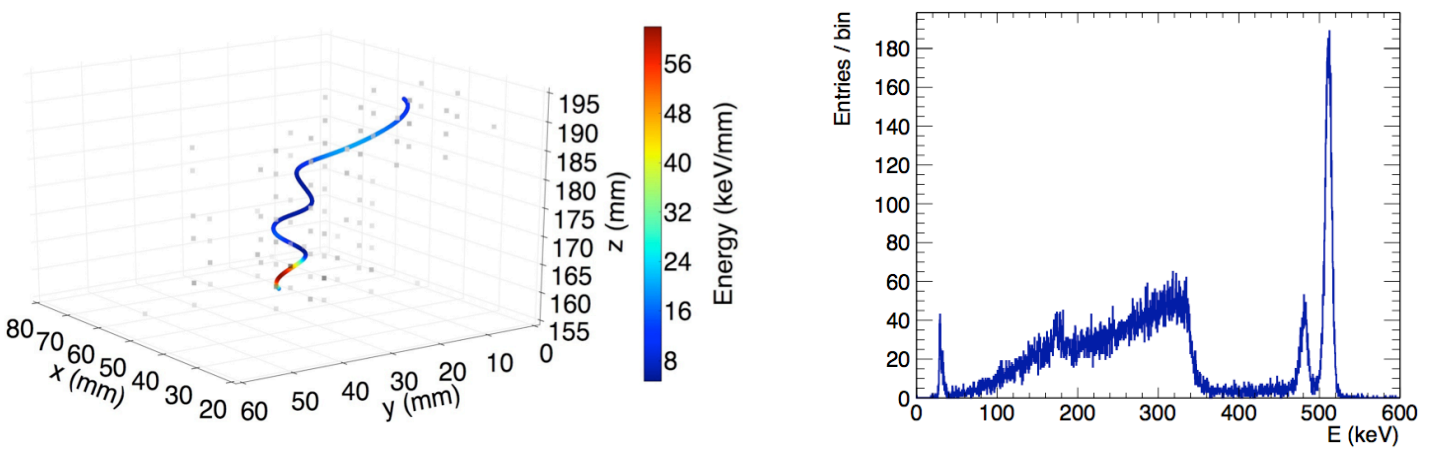

Figure 3. Left: Track reconstruction of an electron ${ }^{137} \mathrm{Cs}$ photo-produced. Right: Energy spectra for ${ }^{22} \mathrm{Na}$ gamma-ray events.

Different types of radiation have been used in NEXT-DEMO to get information: photons (from the decay of the ${ }^{22} \mathrm{Na}$ and ${ }^{137} \mathrm{Cs}$ isotopes), alpha particles (from ${ }^{226} \mathrm{Ra}$ decay) and cosmic muons. As can be see in the Fig. 3, the tracking plane can reconstruct the track of an electron photo- 
produced $(662 \mathrm{keV})$ showing the largest energy deposition at the end of the track. Also, the energy resolution is excellent, with FWHM $=1.5 \%\left(0.78 \%\right.$ at $\left.\mathrm{Q}_{\beta \beta}\right)$, as required in NEXT-100.

\subsection{Prototype NEXT-DBDM}

NEXT-DBDM [5] is a prototype built at the Lawrence Berkeley National Laboratory (CA, USA). It is also a high-pressure (10 - $\left.15 \mathrm{~atm}, \rho=5 \cdot 10^{-2} \mathrm{~g} / \mathrm{cm}^{3}\right)$ xenon electroluminescent TPC with 19 Hamamatsu R7378A PMTs and 64 TPB coated SiPMs. Among its main objectives is to demonstrate near-intrinsic resolution at energies up to $662 \mathrm{keV}$. Energy resolutions of 1\% FWHM for $662 \mathrm{keV}$ gamma rays were obtained at 10 and 15 atm and 5\% FWHM for $30 \mathrm{keV}$ fluorescence (Fig. 4).
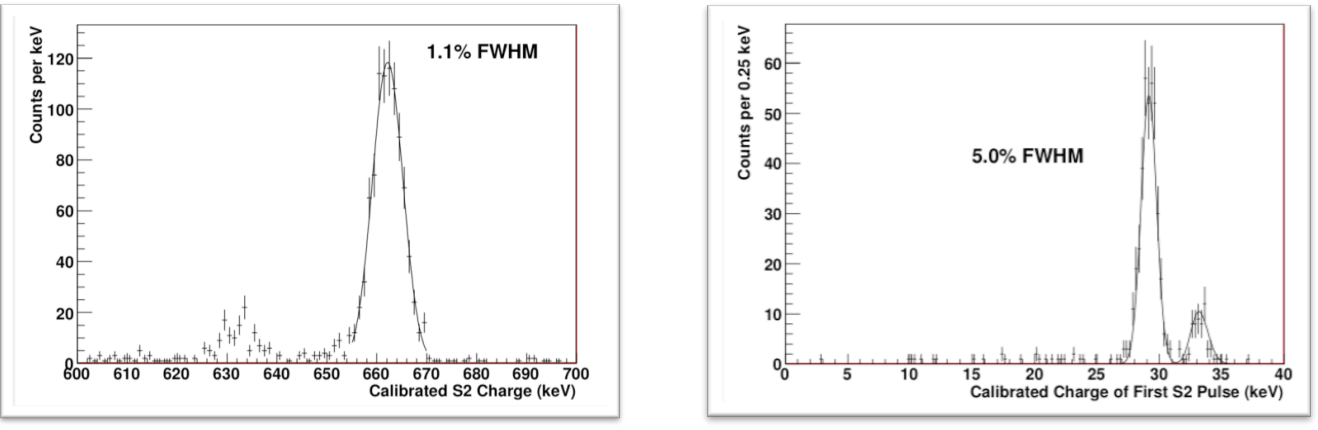

Figure 4 Left: $662 \mathrm{keV}$ gamma peak $\left(0.57 \%\right.$ FWHM at $\left.\mathrm{Q}_{\beta \beta}\right)$. Right: X-ray peaks (K-alpha and K-beta)

\subsection{NEXT-100}

NEXT-100 (100kg of ${ }^{136} \mathrm{Xe}$ at $\left.15 \mathrm{bar}\right)$ is now in the construction process, scheduled to start in 2015. The main platform where the detector will be placed is already mounted at the LSC. The vessel is almost built, all the photosensors have been already purchased and a significant fraction of the electronic components are in construction and testing process. Different components are waiting to its scheduled radio-purity measurement in order to validate them.

\section{Radiopurity}

In NEXT, only a few events per year are expected and this implies a careful screening and selection of the most radiopure materials to be used in the experiment and decrease the intrinsic background. To perform the radiopurity measurements we use mainly "High Purity Germanium detectors (HP-Ge)". Other techniques like "Glow-Discharge Mass Spectrometry (GDMS)" have also been used.
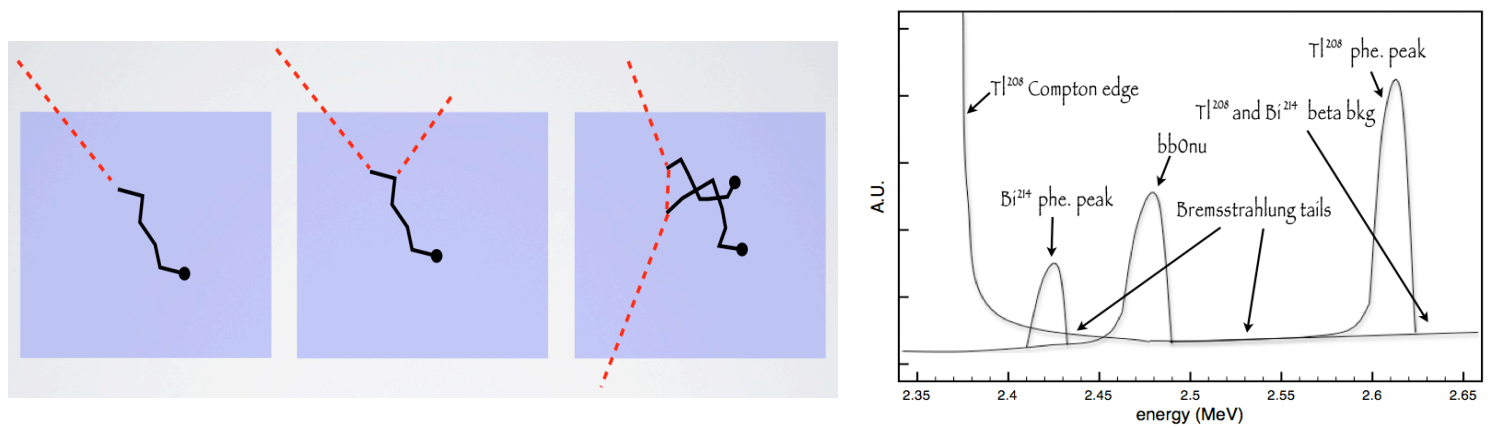

Figure 5. Left: 3 cases of dangerous background events. Right: NEXT-100 ROI 


\subsection{Background Model}

The most dangerous background events for NEXT (see Fig. 5) are: case 1, electron photoproduced by $2448 \mathrm{keV}$ gamma from ${ }^{214} \mathrm{Bi}$ decay; case 2, electron photo-produced by $2615 \mathrm{keV}$ gamma from ${ }^{208} \mathrm{Tl}$ decay that undergoes Bremsstrahlung and case 3, two electron Compton scattered from $2615 \mathrm{keV}$ gamma from ${ }^{208} \mathrm{Tl}$ decay.

Preliminary estimations from our background model impose the following limits to the radioactive contaminations $0.18-0.40 \cdot 10^{-3}$ counts $/ \mathrm{kg} / \mathrm{keV} /$ year for ${ }^{214} \mathrm{Bi}$ and $0.21-0.48 \cdot 10^{-3}$ counts $/ \mathrm{kg} / \mathrm{keV} /$ year for ${ }^{208} \mathrm{Tl}$. Those limits force us to carry out an intense radiopurity campaign to select suficiently radiopure materials for our detector.

\subsection{LSC facilites}

Canfranc Underground Laboratory (LSC, Laboratorio Subterráneo de Canfranc) is located in the Spanish side of the Pyrenees, under the Tobazo mountain, with approximately 2450 m.w.e. overburden that suppresses the cosmic muon flux by approximately 5 orders of magnitude. The radon activity in the air ranges from 50 to $80 \mathrm{~Bq} / \mathrm{m}^{3}$. The LSC offers a Radiopurity Service to measure ultra-low radioactivity using four high purity germanium detectors (this number will be increasing gradually).

\subsubsection{HP-Ge detectors}

The four HP-Ge detectors used are p-type, close-end, coaxial from Canberra Industries Inc. The active volume of the crystal is $410-420 \mathrm{~cm}^{3}$ (the cryostat made of ultra-low background aluminum) with $100-110 \%$ relative efficiencies and FWHM $\approx 2 \mathrm{keV}(1332 \mathrm{keV})$. The detector is shielded with a $5 \mathrm{~cm}$ thick copper layer followed by a $20 \mathrm{~cm}$ thick layer. In addition, the setup is inside a nitrogen pressurized methacrylate box to avoid radon intrusion.

Fig. 6 shows the background spectrum of one of the LSC detectors, geOroel. It amounts approximately 500 counts/day between $100-2700 \mathrm{keV}$. The relevant gamma lines of $583 \mathrm{keV}$ $\left({ }^{208} \mathrm{Tl}\right)$ and $609 \mathrm{keV}\left({ }^{214} \mathrm{Bi}\right)$ contribute with 1 and 3 counts per day respectively. To know the value of the activity or to estimate an upper limit, we follow Gator facility criteria [6].

\subsection{Screening program}

The screening program of NEXT [7] [8] is in a rather advance state. An example of result, among the most relevant obtained so far, is that of the 316Ti stainless steel supplied by the Nironit company, of the order of tenths of $\mathrm{mBq} / \mathrm{kg}$ for the isotopes at the lower part of the ${ }^{238} \mathrm{U}$ and ${ }^{232}$ Th chains.

Several important measurements are being done or yet awaiting for measurement time. As an almost independent effort is the screening campaign of the photomultiplier composing the energy plane.

\subsection{PMT campaign}

In the energy plane, 60 Hamamatsu R11410-10 3-inch photomultipliers (Fig. 7, left) will be placed, that are possibly one of our largest source of background for the $2 \beta 0 \mathrm{v}$ signal we are looking for. These PMTs are the first low background-designed commercial PMTs, thus their measurements are expected to provide very valuable information to the low background 
community. In addition, a comparison to previous measurements by LUX ( $\approx 0.7 \mathrm{mBq} / \mathrm{PMT})[9]$ and XENON $(\approx 5 \mathrm{mBq} / \mathrm{PMT})$ [10] Collaborations, will give important information about reproducibility in the fabrication process and explain this difference.

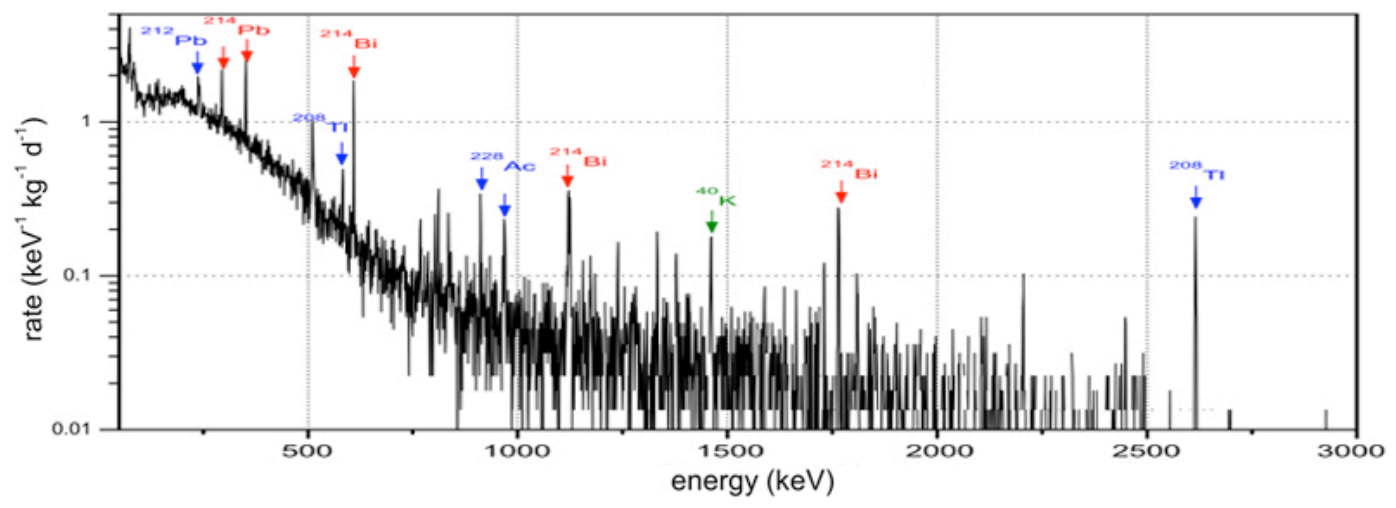

Figure 6. Background spectrum of geOroel detector

Because of the large size of the sample and the limited measurement time, all the PMTs in a mode pass/don't pass are being measured at the moment. The idea is to identify PMTs with high activity (activities larger than $5 \mathrm{mBq}$ in either isotope are identified within few days), making short measurements with 3 PMTs simultaneously (Fig. 7, right). Once this phase is finished, we will proceed with precision measurements of individual PMTs.
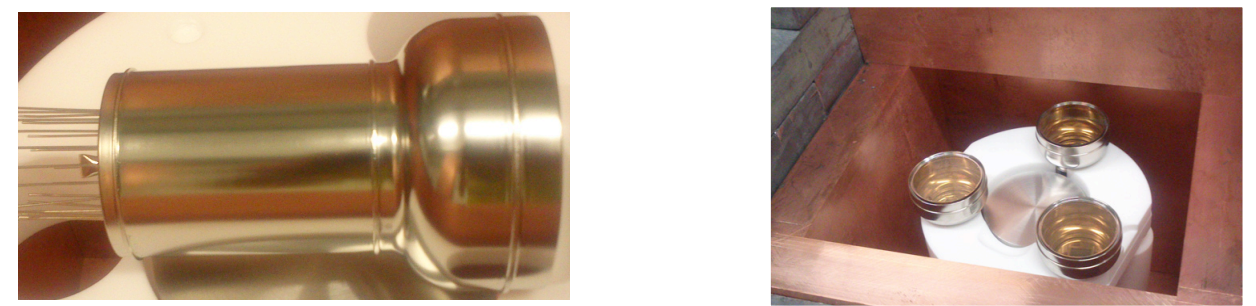

Figure 7. Left: PMT R11410-10. Right: PMTs and HP-Ge detector.

GEANT 4.9.5 Monte Carlo simulations are being used to estimate the detection efficiencies (2\% typically at $600 \mathrm{keV}$ level). We have already performed 7 measurements of 3 PMTs and one initial of a single PMT. The measurement time is around 20 days.

Table. 1 shows the results of these measurements. The measured activities are well within expectations and appropriate for the experiment. We expect to finish this pass/don't-pass phase by spring of 2014. Afterwards we will proceed with high precision measurements of as many PMTs units as the time period until the start up of the experiments permits.

\begin{tabular}{|c|c|c|c|c|c|c|}
\hline & 3РMT01 & 3РMT02 & 3РMT03 & 3РMT04 & 3РMT05 & 3РMT06 \\
\hline Time [days] & 30.64 & 20.22 & 16.57 & 18.31 & 40.11 & 36.94 \\
\hline${ }^{232} \mathrm{Th}$ Chain $\left({ }^{208} \mathrm{Tl}\right)[\mathrm{mBq}]$ & $<7.1$ & $<9.2$ & $<8.8$ & $<7.9$ & $<7.1$ & $<6.2$ \\
\hline${ }^{232} \mathrm{Th}$ Chain $\left({ }^{228} \mathrm{Ac}\right)[\mathrm{mBq}]$ & $<9.5$ & $<11.1$ & $<11.0$ & $<11.0$ & $<7.5$ & $<8.4$ \\
\hline${ }^{238} \mathrm{U}$ Chain $\left({ }^{214} \mathrm{Bi}\right)[\mathrm{mBq}]$ & $<3.2$ & $<3.9$ & $<4.2$ & $<5.0$ & $<3.3$ & $<2.7$ \\
\hline
\end{tabular}




\begin{tabular}{|c|c|c|c|c|c|c|}
\hline${ }^{238} \mathrm{U}$ Chain $\left({ }^{234} \mathrm{~Pa}\right)[\mathrm{mBq}]$ & $<329$ & $<420$ & $<610$ & $<386$ & $<248$ & $<269$ \\
\hline${ }^{40} \mathrm{~K}[\mathrm{mBq}]$ & $37.2 \pm 9.9$ & $<73$ & $<58$ & $<68$ & $35.1 \pm 16.9$ & $31.0 \pm 16.6$ \\
\hline${ }^{60} \mathrm{Co}[\mathrm{mBq}]$ & $15.3 \pm 1.1$ & $13.3 \pm 1.1$ & $12.0 \pm 1.0$ & $13.1 \pm 1.4$ & $13.5 \pm 1.0$ & $12.0 \pm 0.9$ \\
\hline${ }^{54} \mathrm{Mn}[\mathrm{mBq}]$ & $1.40 \pm 0.35$ & $<2.1$ & $<2.2$ & $<1.6$ & $1.4 \pm 0.5$ & $1.0 \pm 0.5$ \\
\hline
\end{tabular}

Table 1. Preliminary results of PMTs activities

4.Summary

NEXT is an excellent option for this generation of $2 \beta 0 \mathrm{v}$ experiments, thanks to its good energy resolution, its ability to reject background by tracking and its scalability to larger detector volumes.

The results from the NEXT-DBDM and NEXT-DEMO prototypes are extremely important milestones. They have proven the technology, the energy resolution and tracking with different gamma sources.

A thorough screening program is being carried out at the LSC. The materials chosen for NEXT are showing good radio-purity properties. A special campaign for the 60 low radioactivity PMTs is also ongoing with a pass/don't-pass selection criterion. The units measured so far show very small radioactive contamination that makes them valid for the experiment.

References

[1] The NEXT Collaboration, NEXT-100 Technical Design Report; Executive Summary 2012 JINST 7 T06001

[2] Bolotnikov et al., The spectroscopic propierties of high-pressure xenon, NIM A 396 (1997) 360$370 \mathrm{~A}$

[3] The NEXT Collaboration, Initial Results of NEXT-DEMO, a Large-scale Prototype of the NEXT-100 Experiment, 2013 JINST 8 P04002

[4] The NEXT Collaboration, Operation and First Results of the NEXT-DEMO Prototype using a Silicon Photomultiplier Tracking Array, arXiv:1306.0471

[5] The NEXT Collaboration, Near-Intrinsic Energy Resolution for 30 to $662 \mathrm{keV} \mathrm{Gamma} \mathrm{Rays} \mathrm{in} \mathrm{a}$ High Pressure Xenon Electroluminescent TPC, Nucl. Inst. Meth. A708 (2013) 101

[6] L. Baudis et al., Gator: a low-background counting facility at the Gran Sasso Underground Laboratory, JINST 6 (2011) P08010

[7] The NEXT Collaboration, Radiopurity control in the NEXT-100 double $\beta$ decay experiment: procedures and initial measurements. 2013 JINST 8 T01002

[8] The NEXT Collaboration, Radiopurity control in the NEXT-100 double beta decay experiment, AIP Conf. Proc. 1549 (2013) 46-49

[9] D. Akerib et al., An Ultra-Low Background PMT for Liquid Xenon Detectors, NIM A 703 (2013) $1-6$

[10]E. Aprile et al., Material screening and selection for XENON100, Astropart. Phys, 35 (2011) 4349 